\title{
ADESÃO À PRÁTICA DE EXERCÍCIOS PARA REABILITAÇÃO FUNCIONAL DE MULHERES COM CÂNCER DE MAMA: REVISÃO DE LITERATURA
}

\author{
ADHERENCE TO THE PRACTICE OF EXERCISES FOR FUNCTIONAL \\ REHABILITATION OF WOMEN WITH BREAST CANCER: \\ A LITERATURE REVIEW
}

\section{ADHERENCIA A LA PRÁCTICA DE EJERCICIOS PARA REHABILITACIÓN FUNCIONAL DE MUJERES CON CÁNCER DE MAMA: REVISIÓN DE LITERATURA}

\author{
DinORAH Kovacs SANT'ANNA* \\ VANESSA DE AlmeIdA ${ }^{*}$ \\ Eliana LouZada Petito ${ }^{* * *}$ \\ Maria Gaby Rivero de GutiérReZ ${ }^{* * * *}$
}

\begin{abstract}
RESUMO
Introdução: O tratamento cirúrgico para o câncer de mama pode implicar em complicações pós-operatórias, como diminuição da amplitude de movimento e da funcionalidade do membro superior. Objetivos: identificar estudos sobre adesão a programas de reabilitação funcional, descrever os instrumentos utilizados para avaliá-la e identificar os fatores que favorecem ou dificultam esse comportamento. Método: Revisão de literatura nas bases de dados PUBMED, MEDLINE e LILACS, no período de 1997 a 2008, utilizando os termos: neoplasia, mama, exercício, reabilitação, aderência. Resultados: Dos 52 artigos encontrados, apenas oito se referiam à adesão a exercícios, escalas e instrumentos para mensurá-la. Os diários com o auto-relatos das pacientes foram os instrumentos mais utilizados. A adesão é influenciada de forma positiva por fatores como percepção dos benefícios, envolvimento dos profissionais e da família e participação em grupos de apoio. A falta de tempo, os efeitos colaterais dos tratamentos antineoplásicos, os afazeres domésticos e as responsabilidades familiares constituem as principais barreiras ao seguimento do programa de exercícios. Conclusão: A constância e a regularidade na prática de exercícios são enfatizadas para a obtenção de resultados positivos na reabilitação. Avaliar a adesão implica na observação de vários fatores que vão além do comparecimento às sessões. São necessárias ações que reforcem os aspectos facilitadores e atenuem as barreiras, aproximando as pacientes dos profissionais de saúde.
\end{abstract}

Palavras chave: Neoplasia, mama, exercício, reabilitação, aderência.

\begin{abstract}
Introduction: Surgical treatment for breast cancer may lead to post-operative complications such as the diminution of the range of movement and the functionality of the arm. Objectives: To identify studies related to the adherence to functional rehabilitation programs, to describe the instruments that were used to evaluate the adherence and the factors that can facilitate or make it difficult. Methods: Literature review in the PUBMED, MEDLINE and LILACS databases, searching the period from 1997 to 2008, using the combined terms: breast, neoplasm, exercise, rehabilitation and adherence. Findings: From the 52 papers found, only 8 were related to

\footnotetext{
*Aluna de graduação em Enfermagem, EPE/UNIFESP, Brasil. Email: dinorahkovacs@hotmail.com

${ }^{* *}$ Aluna de graduação em Enfermagem, EPE/UNIFESP, Brasil. Email: vanescau@hotmail.com

${ }^{* * *}$ Aluna de Mestrado do Programa de Pós-Graduação em Enfermagem - EPE/UNIFESP, Brasil. Email: lica.sp@ig.com.br

${ }^{* * * *}$ Orientadora e docente da EPE/UNIFESP, Brasil. Email: mggutierrez@denf.epm.br
} 
the adherence to the exercises practice as well as to scales and tools to measure it. The diaries with auto report by the patients were the more used instruments. The adhesion is favored by factors such as the perception of benefits, the health professionals and family involvement and the participation at support groups. And that the lack of available time, the side effects of antineoplastic treatments and the home and family tasks are the main factors that difficult to continuing the exercises program. Conclusion: Constance and regularity in the exercises practice is emphasized for achieving positive results in rehabilitation. Evaluate adherence to it refers to the observation of many factors, not only the frequency. There are actions that are necessary in order to reinforce the facilitating aspects and to diminish the barriers, approaching patients and health professionals.

Key words: Neoplasm, breast, exercise, rehabilitation, adherence.

\section{RESUMEN}

Introducción: El tratamiento quirúrgico para el cáncer de mama puede ocasionar complicaciones pos-operatorias como la reducción de la amplitud del movimiento y de la funcionalidad del miembro superior. Objetivos: Identificar estudios sobre la adherencia a la práctica de ejercicios físicos o a programas de rehabilitación funcional, describir los instrumentos utilizados para evaluarla e identificar los factores que favorecen o dificultan ese comportamiento. Método: Revisión de literatura en las bases de datos Pubmed, Medline y Lilacs, en el período de 1997 a 2008, utilizando los términos: neoplasia, mama, ejercicio, rehabilitación y adherencia. Resultados: De los 52 artículos encontrados, apenas ocho se referían a la adhesión a los ejercicios, a escalas e instrumentos para mensurarla. Los diarios con el autorrelato de las pacientes fueron los instrumentos más utilizados. La adherencia es influenciada de forma positiva por factores como percepción de los beneficios, involucración de los profesionales y de la familia y la participación en grupos de apoyo. La falta de tiempo, los efectos colaterales de los tratamientos, los quehaceres domésticos y las responsabilidades familiares constituyen las principales barreras para el seguimiento del programa de ejercicios. Conclusión: Se da énfasis a la constancia y regularidad en la práctica de los ejercicios para la obtención de resultados positivos en la rehabilitación. Evaluar la adherencia implica la observación de varios factores que van más allá de la comparecencia a las sesiones. Son necesarias acciones que refuercen los aspectos facilitadores y atenúen las barreras, aproximando las pacientes de los profesionales de la salud.

Palabras clave: Neoplasia, mama, ejercicio, rehabilitación, adherencia.

Fecha recepción:10/04/08 Fecha aceptación: 03/12/09

\section{INTRODUÇÃO}

O câncer de mama é o segundo tipo de câncer mais freqüente no mundo. Possui bom prognóstico quando detectado precocemente, especialmente pelo exame clínico das mamas e da mamografia, e quando instituído tratamento adequado. A indicação das diferentes modalidades terapêuticas depende do estadiamento clínico e do tipo histológico do tumor (1).

As modalidades terapêuticas para o câncer de mama são: cirurgia e radioterapia para tratamento loco-regional, e quimioterapia e hormonioterapia para tratamento sistêmi- co. A cirurgia pode ter caráter conservador, como a tumorectomia, a setorectomia e a quadrantectomia onde há ressecção de um segmento da mama com possível retirada dos gânglios axilares ou linfonodo sentinela, ou não conservador como a mastectomia que consiste na retirada de toda a mama (2).

O tratamento cirúrgico freqüentemente gera co-morbidades que causam grande temor entre as mulheres, provocando alterações físicas, como desconforto e déficits da motricidade; psicológicas, que podem afetar a sexualidade e a imagem pessoal; e sociais, como alterações nas relações familiares e preconceito $(3,4)$.

Quanto maior a extensão da cirurgia, bem 
como a realização do esvaziamento axilar, maior a possibilidade de complicações como linfedema, dor, parestesias, diminuição da força muscular e redução da amplitude de movimento (ADM) do membro envolvido, merecendo atenção por estarem diretamente relacionados com o retorno às atividades da vida diária das pacientes, bem como com a qualidade de vida destas.

Nesse sentido, a prática de exercícios após intervenções cirúrgicas tem fundamental importância na recuperação da mobilidade e amplitude de movimentos, prevenindo ou minimizando a atrofia de músculos e limitações articulares, e na tentativa de redução do surgimento de linfedema (5).

A mobilização do ombro, quando realizada precocemente, auxilia no restabelecimento da função e desperta o sentimento de independência, ajudando a estimular a mulher a prosseguir com o programa de exercícios, parte integrante do tratamento.

A partir da década de 70 começou-se a utilizar programas de exercícios na reabilitação, sendo que atualmente se observa uma tendência preventiva, sobrepondo-se ao caráter reabilitativo puro e simples, objetivando minimizar a ocorrência de complicações (6).

A prática de exercícios para reabilitação do membro homolateral à cirurgia do câncer de mama, assim como as demais terapêuticas de longo prazo, implica na necessidade da adesão da paciente ao regime de tratamento, bem como no incentivo e apoio da família e da equipe de saúde para o alcance dos objetivos.

A adesão é um importante indicador da efetividade do sistema de saúde, e a não adesão é classificada como um problema causado pela interação de quatro fatores que possuem influência recíproca no processo de cuidado de doenças crônicas, a saber: a equipe de saúde e fatores relacionados ao sistema, fatores relacionados às circunstâncias, características da terapia e fatores relacionados ao paciente (7).

Não há consenso sobre a definição de adesão, nem mesmo como mensurá-la, prin- cipalmente em se tratando de programas de exercícios domiciliares, onde não se tem a presença e supervisão direta das pacientes na prática diária dos exercícios recomendados.

$\mathrm{Na}$ literatura encontram-se diversas definições para adesão, entre elas: o quanto o paciente segue as instruções do profissional de saúde (7), o quanto o comportamento do paciente coincide com as recomendações médicas $(8,9)$, o comprometimento do paciente frente às recomendações $(4,10) \mathrm{e}$, especificamente para os programas de exercícios na reabilitação de mulheres mastectomizadas, foi descrita a recomendação de um comparecimento às sessões de exercícios de aproximadamente $70 \%$ das sessões propostas $(11,12,13)$. Autores mencionam, ainda, o fator psicológico que pode influenciar de forma negativa ou positiva a adesão ao programa de exercícios (14).

No que se refere à avaliação da adesão de mulheres a um programa de reabilitação funcional, poucas fontes bibliográficas foram encontradas $(11,12,13,15,16)$. A maior parte dos estudos sobre adesão se faz sobre terapias medicamentosas de doenças crônicas, o que evidencia a necessidade de desenvolver instrumentos específicos destinados à avaliação da prática de exercícios.

Considerando a reabilitação fruto de um processo de desenvolvimento de práticas físicas e comportamentais, julga-se imprescindível a participação integral da paciente em todo o contexto de sua realização, de modo a atingir o objetivo final de proporcionar-lhe condições de realizar as atividades da vida diária, sem perder de vista a qualidade de vida dessa mulher.

Diante do exposto, os objetivos desse estudo foram: identificar, na literatura, estudos que abordam a adesão à prática de exercícios físicos ou a programas de reabilitação pósoperatória de cirurgia oncológica de mama; descrever os instrumentos utilizados para avaliar a adesão a programas de reabilitação; explorar as dificuldades e facilidades que influenciam a adesão a esse tipo de programa. 
MÉTODO

Revisão de literatura nas bases de dados LILACS, PUBMED e MEDLINE, compreendendo o período de 1997 a 2008, utilizando os descritores: neoplasia, mama, exercício, reabilitação, aderência.

A partir da combinação desses descritores, foram localizados 52 artigos (PUBMED: 26; MEDLINE: 24; LILACS: 2). Desse total, 44 faziam referência a aspectos conceituais ou à qualidade de vida, não englobando os objetivos principais do estudo.

\section{RESULTADOS}

Os oito trabalhos selecionados incluíam os fatores que influenciavam a adesão de mulheres com câncer de mama à prática de exercícios ou programas de reabilitação(4, $11,13,14)$, e também a forma de avaliação dessa adesão(11, 12, 13-17).

Com relação aos fatores relacionados à adesão, estes eram tanto facilitadores quanto dificultadores, influenciando de forma positiva ou negativa na adesão à realização dos exercícios, conforme descrito no Tabela 1.

Tabela 1. Fatores que favorecem ou dificultam a adesão a programas de exercícios para reabilitação funcional. São Paulo, 2008.

\begin{tabular}{|c|c|c|c|}
\hline \multirow[t]{2}{*}{ AUTOR / ANO } & \multirow[t]{2}{*}{ OBJETIVO } & \multicolumn{2}{|l|}{ FATORES } \\
\hline & & FAVORECEM & DIFICULTAM \\
\hline $\begin{array}{l}\text { Prado et al. } 2004(4) \\
\text { A prática da atividade } \\
\text { física em mulheres } \\
\text { submetidas à cirurgia } \\
\text { por câncer de } \\
\text { mama: percepção de } \\
\text { barreiras e benefícios }\end{array}$ & $\begin{array}{l}\text { - verificar a adesão á atividade } \\
\text { física identificando as barreiras } \\
\text { e incentivos a esta prática em } \\
\text { mulheres com câncer de mama }\end{array}$ & $\begin{array}{l}\text { - melhora da condição física: } \\
\text { fortalecimento muscular, do } \\
\text { movimento dos braços, sensação de } \\
\text { prazer e disposição } \\
\text { - conhecimento sobre a importância } \\
\text { da prática dos exercícios } \\
\text { - acompanhamento profissional } \\
\text { - suporte de familiares }\end{array}$ & $\begin{array}{l}\text { - falta de força de } \\
\text { vontade } \\
\text { - falta de tempo } \\
\text { - cansaço }\end{array}$ \\
\hline $\begin{array}{l}\text { Daley et al. } 2007(11) \\
\text { Determinants of } \\
\text { adherence to exercise } \\
\text { in women treated of } \\
\text { breast cancer }\end{array}$ & $\begin{array}{l}\text { - verificar fatores facilitadores } \\
\text { e barreiras à adesão a dois } \\
\text { programas de exercícios e cuidados } \\
\text { usuais em pacientes com câncer } \\
\text { de mama } \\
\text { - adesão } \geq 70 \% \text { comparecimento } \\
\text { às sessões }\end{array}$ & $\begin{array}{l}\text { - horário livre para a realização dos } \\
\text { exercícios } \\
\text { - acompanhamento profissional } \\
\text { - envolvimento da família }\end{array}$ & $\begin{array}{l}\text { - afazeres domésticos / } \\
\text { ocupações familiares } \\
\text { - compromisso de } \\
\text { trabalho } \\
\text { - duração e tipo de } \\
\text { tratamento }\end{array}$ \\
\hline $\begin{array}{l}\text { Ott et al. 2004(14) } \\
\text { Facilitative strategies } \\
\text { psychological factors, } \\
\text { and strength/weigth } \\
\text { training behaviors } \\
\text { in breast cancer } \\
\text { survivors who are at } \\
\text { risk for osteoporosis }\end{array}$ & $\begin{array}{l}\text { - verificar os fatores psicológicos } \\
\text { e relacioná-los com a adesão ou } \\
\text { não a um programa de treinamento } \\
\text { de resistência para mulheres com } \\
\text { câncer de mama }\end{array}$ & $\begin{array}{l}\text { - informação e percepção dos } \\
\text { benefícios } \\
\text { - acompanhamento profissional } \\
\text { - compromisso na realização dos } \\
\text { exercícios }\end{array}$ & $\begin{array}{l}\text { - não relaciona as } \\
\text { barreiras encontradas } \\
\text { à prática de exercícios }\end{array}$ \\
\hline $\begin{array}{l}\text { Roger et al. 2007(15) } \\
\text { Exercise stage of } \\
\text { change, barriers, } \\
\text { expectations, values } \\
\text { and preferences } \\
\text { among breast cancer } \\
\text { patients during } \\
\text { treatment: a pilot } \\
\text { study }\end{array}$ & $\begin{array}{l}\text { - determinar as barreiras à prática } \\
\text { de exercício físico e a preferência } \\
\text { de tipos exercícios em pacientes } \\
\text { com câncer de mama por meio do } \\
\text { uso de questionário }\end{array}$ & $\begin{array}{l}\text { - percepção dos benefícios } \\
\text { do exercício sobre o sistema } \\
\text { cardiovascular, aumento de força } \\
\text { muscular, perda de peso }\end{array}$ & $\begin{array}{l}\text { - falta de disciplina } \\
\text { - falta de tempo } \\
\text { - falta de interesse } \\
\text { - efeitos colaterais } \\
\text { da QT } \\
\text { - desânimo } \\
\text { - afazeres domésticos } \\
\text { / ocupações familiares } \\
\text { - compromisso de } \\
\text { trabalho }\end{array}$ \\
\hline
\end{tabular}




\begin{tabular}{|c|c|c|c|}
\hline $\begin{array}{l}\text { Gutiérrez et al. } \\
2007(17) \\
\text { Adesão de mulheres } \\
\text { mastectomizadas } \\
\text { ao início precoce } \\
\text { de um programa de } \\
\text { reabilitação }\end{array}$ & $\begin{array}{l}\text { - verificar a adesão a um programa } \\
\text { de reabilitação precoce em } \\
\text { pacientes com câncer de mama } \\
\text { - identificar as dificuldades na } \\
\text { realização dos exercícios e das } \\
\text { atividades de vida diária }\end{array}$ & $\begin{array}{l}\text { - presença de profissionais } \\
\text { especializados } \\
\text { - grupos de apoio } \\
\text { - suporte familiar } \\
\text { - reforço das orientações por meio } \\
\text { de material ilustrativo }\end{array}$ & $\begin{array}{l}\text { - dor, ardência } \\
\text { - medo de afetar a } \\
\text { cirurgia } \\
\text { - repuxo } \\
\text { - sensação de peso } \\
\text { - fraqueza e mal-estar } \\
\text { - falta de sensibilidade } \\
\text { na região da cirurgia } \\
\text { e mãos } \\
\text { - falta de vontade }\end{array}$ \\
\hline $\begin{array}{l}\text { Rogers et al. } \\
2006(16) \\
\text { Exercise barrier and } \\
\text { task self-efficacy in } \\
\text { breast cancer patients } \\
\text { during treatment }\end{array}$ & $\begin{array}{l}\text { - mensurar barreiras ao exercício } \\
\text { e auto-eficiência, por meio de } \\
\text { escalas } \\
\text { - observar associações entre } \\
\text { barreiras e auto-eficiência, entre } \\
\text { auto-eficiência e idade, raça e tipo } \\
\text { de tratamento em mulheres com } \\
\text { câncer de mama }\end{array}$ & $\begin{array}{l}\text { - programa de exercícios com } \\
\text { intensidade gradual } \\
\text { - educação/supervisão profissional } \\
\text { - supervisão médica de co- } \\
\text { morbidades }\end{array}$ & $\begin{array}{l}\text { - náusea } \\
\text { - fadiga } \\
\text { - falta de tempo } \\
\text { - falta de apoio } \\
\text { - cansaço } \\
\text { - falta de interesse } \\
\text { - falta de prazer } \\
\text { na realização dos } \\
\text { exercícios }\end{array}$ \\
\hline $\begin{array}{l}\text { Courneya et al. } \\
\text { 2008(12) } \\
\text { Barriers to supervised } \\
\text { exercise training } \\
\text { in a randomized } \\
\text { controlled trial } \\
\text { of breast cancer } \\
\text { patients receiving } \\
\text { chemotherapy }\end{array}$ & $\begin{array}{l}\text { - relatar as barreiras aos exercícios } \\
\text { supervisionados para mulheres } \\
\text { com câncer de mama }\end{array}$ & & $\begin{array}{l}\text { - sentir-se doente } \\
\text { - efeitos colaterais QT } \\
\text { - falta interesse } \\
\text { - dor } \\
\text { - trabalho } \\
\text { - dificuldades de } \\
\text { transporte } \\
\text { - férias }\end{array}$ \\
\hline $\begin{array}{l}\text { Courneya et al. } \\
\text { 2008(13) } \\
\text { Predictors of } \\
\text { supervised exercise } \\
\text { adherence during } \\
\text { breast cancer } \\
\text { chemotherapy }\end{array}$ & $\begin{array}{l}\text { - relatar os preditores à aderência } \\
\text { aos exercícios supervisionados } \\
\text { para mulheres com câncer de } \\
\text { mama }\end{array}$ & $\begin{array}{l}\text { - supervisão individual } \\
\text { - maior flexibilidade de horário do } \\
\text { local de realização dos exercícios } \\
\text { - experiência anterior com } \\
\text { exercícios } \\
\text { - menor depressão } \\
\text { - estágios mais avançados } \\
\text { - maior nível de escolaridade }\end{array}$ & $\begin{array}{l}\text { - efeitos colaterais do } \\
\text { tratamento }\end{array}$ \\
\hline
\end{tabular}

QT= quimioterapia

No que se refere à utilização de algum tipo de instrumento para avaliar a adesão a programas de reabilitação, identificou-se na busca bibliográfica seis artigos que utilizaram instrumentos, como questionários ou diários, alguns dos quais eram preenchidos pelo próprio paciente e outros pelos profissionais de saúde $(11,12,13-17)$, conforme o Tabela 2.

Tabela 2. Formas de avaliação da adesão a programas de exercícios. São Paulo, 2008.

\begin{tabular}{|c|l|}
\hline \multicolumn{2}{|c|}{ Autores } \\
\hline $\begin{array}{c}\text { Daley et al. 2007(11) } \\
\begin{array}{c}\text { Determinants of adherence to exercise in } \\
\text { women treated of breast cancer }\end{array}\end{array}$ & $\begin{array}{l}\text { - preenchimento de diário de realização dos exercícios pelos pesquisadores } \\
\text { - itens avaliados no diário: intensidade, duração, freqüência e tipo de exercício } \\
\text { realizado durante um período de 8 semanas }\end{array}$ \\
$\begin{array}{c}\text { Ott et al. 2004(14) } \\
\text { Facilitative strategies psychological factors, } \\
\text { and strength/weigth training behaviors in } \\
\text { breast cancer survivors who are at risk for } \\
\text { osteoporosis }\end{array}$ & $\begin{array}{l}\text { - uso de caderno para registro da freqüência, repetições, séries e dúvidas ou } \\
\text { pelas enfermeiras; } \\
\text { - cálculo da adesão mensal (no de realizações referido pelas pacientes/no } \\
\text { mensal) }\end{array}$ \\
\hline
\end{tabular}




\begin{tabular}{|c|c|}
\hline $\begin{array}{c}\text { Rogers et al. 2007(15) } \\
\text { Exercise stage of change, barriers, } \\
\text { expectations, values and preferences among } \\
\text { breast cancer patients during treatment: a } \\
\text { pilot study }\end{array}$ & $\begin{array}{l}\text { - resposta a questionário } \\
\text { - classificação conforme algoritmo pré-estabelecido sobre a realização de } \\
\text { exercícios } \\
\text { - participantes classificaram, de acordo com uma escala de } 5 \text { pontos, } \\
\text { quesitos sobre o quanto as barreiras encontradas interferiram na realização } \\
\text { dos exercícios ( } 1=\text { nunca, } 5=\text { muito freqüentemente) e as expectativas dos } \\
\text { resultados incluindo os riscos e benefícios dos exercícios indicados ( } 1= \\
\text { discorda fortemente, } 5=\text { concorda fortemente) }\end{array}$ \\
\hline $\begin{array}{l}\text { Gutiérrez et al. } 2007(17) \\
\text { Adesão de mulheres mastectomizadas } \\
\text { ao início precoce de um programa de } \\
\text { reabilitação }\end{array}$ & $\begin{array}{l}\text { - auto-preenchimento de ficha de seguimento da realização dos exercícios no } \\
\text { domicílio } \\
\text { - itens avaliados na ficha: freqüência e descrição das dificuldades }\end{array}$ \\
\hline $\begin{array}{l}\text { Courneya et al. } 2008(12) \\
\text { Barriers to supervised exercise training in a } \\
\text { randomized controlled trial of breast cancer } \\
\text { patients receiving chemotherapy }\end{array}$ & $\begin{array}{l}\text { - resposta a questionário aberto, em diários (auto-preenchimento) e } \\
\text { chamadas telefônicas }\end{array}$ \\
\hline $\begin{array}{l}\text { Courneya et al. 2008(13) } \\
\text { Predictors of supervised exercise adherence } \\
\text { during breast cancer chemotherapy }\end{array}$ & $\begin{array}{l}\text { - resposta a questionário aberto, em diários (auto-preenchimento) e } \\
\text { chamadas telefônicas }\end{array}$ \\
\hline
\end{tabular}

DISCUSSÃO E CONSIDERAÇÕES FINAIS

Os trabalhos analisados são enfáticos em ressaltar a importância da realização de exercícios em mulheres com câncer de mama, após o início do tratamento, quer seja cirúrgico ou quimioterápico, a fim de minimizar os efeitos deletérios à falta de movimentação do membro superior e o descondicionamento físico, culminado com uma melhora da qualidade de vida da paciente.

Para que o programa de exercícios apresente os efeitos desejados, quer seja como um determinante para a promoção da saúde, ou como prevenção e redução de riscos associados a doenças e ou complicações, é necessário que seja realizado de forma regular e constante. Os benefícios do exercício somente podem ser experimentados quando ocorre a participação regular, e a obtenção de êxito exige adesão das mulheres ao programa proposto (4).

Os fatores determinantes para a adesão aos programas de exercícios são os relacionados principalmente à confiança, adquirida pelo paciente para com o profissional envolvido, conquistada na medida em que o paciente encontra acolhimento e compromisso do profissional no seu processo de reabilitação. Os fatores facilitadores mais citados na literatura pesquisada incluem o acompanhamento profissional $(4,11-13,14,17)$, a percepção dos benefícios $(4,14,15)$ e o envolvimento da família $(4,11,17)$.

Existem, entretanto, fatores que dificultam a realização dos exercícios, como a falta de tempo $(4,12,15-16)$, a falta de interesse (12-15), as condições de saúde (12-17), os efeitos colaterais dos tratamentos, principalmente a quimioterapia $(12,15-16)$, os compromissos de trabalho $(11,12-15)$ e o desânimo $(4,15,16)$.

Como a tomada de decisão é um item que pode ter forte impacto na adesão, é importante que o profissional estabeleça vínculos com as pacientes, discutindo as opções de tratamento, informando os benefícios da realização de exercícios, demandando um determinado tempo para a explicação e correção dos mesmos, ou seja, intervindo na educação da paciente e de seus familiares, os quais têm papel decisivo no incentivo e realização dos exercícios no domicílio $(4,11,15-16)$.

Como o retorno às atividades do cotidiano, quer sejam aos afazeres domésticos ou até mesmo ao trabalho, ocorre, em geral, em curto período de tempo, é importante pen- 
sar nos programas de exercícios como forma de reabilitação física e emocional para a retomada dessas atividades. A realização dos exercícios permitirá uma melhor condição funcional do membro superior e do nível de condicionamento, tanto pela manutenção quanto pelo incremento da amplitude de movimento e força muscular. Além disso, o exercício contribui para o aumento da autoestima e percepção da capacidade funcional das mulheres, que se sentem aptas à realização das atividades por elas desenvolvidas antes da cirurgia $(4,16-17)$.

Cabe ressaltar que se faz necessário o estabelecimento de uma medida de avaliação ou monitoramento da adesão ao programa de exercícios realizado extramuros, seja através do preenchimento de diários, resposta a questionários, ou outro tipo de instrumento que auxilie a paciente e a equipe de saúde a gerenciar o progresso obtido por meio do referido programa.

Valendo-se de tais conceitos e tentando relacioná-los e trazê-los para a prática assistencial, pesquisadores têm apresentado os resultados do uso de instrumentos que mensuram a freqüência de exercícios realizados durante a semana e a duração de cada sessão, tendo como critério para determinar uma boa adesão o comparecimento a 70\% ou mais das sessões propostas (11, 13-16).

A escassez de pesquisas sobre a utilização ou validação de instrumentos para monitorar a adesão de pacientes a programas de exercícios extramuros de reabilitação funcional aponta para a necessidade de desenvolver e testar esses instrumentos, a fim de se implementar ações que reforcem os aspectos facilitadores e atenuem as barreiras.

\section{REFERÊNCIAS}

1. Instituto Nacional do Câncer/INCA. Brasil, Ministério da Saúde. Estimativa 2006. Hallado em: http://www.inca.gov. br/estimativa/2006/ [Acesso em setembro de 2006].

2. Sociedade Brasileira de Mastologia, Sociedade Brasileira de Cancerologia, Sociedade Brasileira de Patologia, Federação Brasileira de Ginecologia e Obstetrícia. Projeto, Diretrizes, Diagnóstico e Tratamento do Câncer de Mama, 2001. Hallado em: http://www.projetodiretrizes.org.br/projeto_diretrizes/024.pdf [Acesso em junho de 2007].

3. Ferreira PCA, Neves NM, Correa RD, Barbosa SD, Paim C, Gomes NF et al. Educação e assistência fisioterapêutica às pacientes pós-cirurgia do câncer de mama. $8^{\circ}$ encontro de extensão da UFMG; In: $8^{\circ}$ Encontro de Extensão da UFMG, 2005, Belo Horizonte. Anais. Belo Horizonte: UFMG/PROEX, 2005.

4. Prado MAS, Mamede MV, Almeida AM, Clapis MJ. A prática da atividade física em mulheres submetidas à cirurgia por câncer de mama: percepção de barreiras e benefícios. Rev Latino-am Enfermagem 2004; 12(3):494-502.

5. Pedroso W, Araújo MB, Stevanato E. Atividade física na prevenção e na reabilitação do câncer. Motriz. set./dez. 2005; Rio Claro, v.11 n.3: p.155-160.

6. Batiston AP, Santiago SM. Fisioterapia e complicações físico-funcionais após tratamento cirúrgico do câncer de mama. Fisioterapia e Pesquisa 2005; 12 (3):30-5.

7. World Health Organization-WHO. Meeting Report. Adherence to long-term therapies: policy for action. Genebra. 4-5 June 2001.

8. Lutfey K. On practices of "good doctoring": reconsidering the relationship between provider roles and patient adherence. Sociology of Health \& Illness 2005; 27(4): 421-447.

9. Horwitz RI, Horwitz SM. Adherence to the treatment and health outcomes. Arch Intern Med. 1993; 153: 1863-18.

10. Saba F. A aderência dos praticantes. A prática do exercício físico em academias. 
São Paulo: Manole, 2001. cap 4, p. 61.

11. Daley AJ et al. Determinants of adherence to exercise in women treated of breast cancer. Oncology Nursing; 2007.

12. Courneya KS, McKenzie DC, Reid RD, Mackey JR, Gelmon K, Friedenreich $\mathrm{CM}$ et al. Barriers to supervised exercise training in a randomized controlled trial of breast cancer patients receiving chemotherapy. Ann Behav Med, 2008; 35(1):116-22.

13. Courneya KS, Segal RJ, Gelmon K, Reid RD, Mackey JR, Friedenreich CM et al. Predictors of supervised exercise adherence during breast cancer chemotherapy. Med Sci Sports Exerc, 2008; 40(6):1180-7.

14. Ott CD et al. Facilitative strategies psychological factors, and strength/weigth training behaviors in breast cancer survi- vors who are at risk for osteoporosis. Orthopaedic Nursing, 2004; 23(1):45-52.

15. Rogers LQ, Courneya KS, Shah P, Dunnington G, Hopkins-Price P. Exercise stage of change, barriers, expectations, values and preferences among breast cancer patients during treatment: a pilot study. European Journal of Cancer Care 2007; 16: 55-66.

16. Rogers LQ, Courneya KS, Verhulst S, Markwell S, Lanzotti V, Shah P. Exercise barrier and task self-efficacy in breast cancer patients during treatment. Support Care Cancer, 2006; 14(1):84-90.

17. Gutiérrez MGR, Bravo MM, Chanes DC, De Vivo MCR, Souza GO. Adesão de mulheres mastectomizadas ao início precoce de um programa de reabilitação. Acta Paul Enf, 2007; 20(3):249-54. 